\title{
Исследование магнитных диодов со слоем GaMnAs, изготовленным методом импульсного лазерного осаждения
}

\author{
(C) Б.Н. Звонков ${ }^{1}$, О.В. Вихрова ${ }^{1, \uparrow, ~ Ю . А . ~ Д а н и л о в ~}{ }^{1}$, М.В. Дорохин ${ }^{1}$, А.В. Кудрин ${ }^{1}$, И.Л. Калентьева ${ }^{1}$, \\ Е.А. Ларионова ${ }^{1}$, В.А. Ковальский ${ }^{2}$, О.А. Солтанович ${ }^{2}$
}

${ }^{1}$ Научно-исследовательский фиизико-технический институт

Нижегородского государственного университета им. Н.И. Лобачевского,

603950 Нижний Новгород, Россия

${ }^{2}$ Институт проблем технологии микроэлектроники и особочистых материалов Российской академии наук, 142432 Черноголовка, Московская область, Россия

ฯ E-mail: vikhrova@nifti.unn.ru

(Получена 15 октября 2018 г. Принята к печати 22 октября 2018 г.)

Представлены результаты экспериментального изучения новой конструкции диодных гетероструктур с ферромагнитными слоями (Ga, Mn)As. Диодные структуры изготовлены сочетанием методов MOCгидридной эпитаксии и импульсного лазерного осаждения и содержат гетеропереходы $(\mathrm{Ga}, \mathrm{Mn}) \mathrm{As} / n$-InGaAs. Исследованы электрические свойства диодов в магнитном поле, приложенном перпендикулярно плоскости $p-n$-перехода. Обнаружено отрицательное магнетосопротивление при температурах $<50 \mathrm{~K}-$ меньше температуры Кюри $(\mathrm{Ga}, \mathrm{Mn}) \mathrm{As}$, связанное с уменьшением рассеяния носителей заряда благодаря наличию ферромагнитного упорядочения и со снижением потенциального барьера на границе (Ga, Mn) As/n-InGaAs. Наблюдаемое отрицательное магнетосопротивление немонотонно зависит от величины прямого смещения с максимумом в области напряжения, близкого к высоте потенциального барьера $p-n$-перехода. Наличие максимума может быть связано с определяющим вкладом спин-зависимых сопротивления слоя (Ga, $\mathrm{Mn}) \mathrm{As}$ и сопротивления на границе раздела $(\mathrm{Ga}, \mathrm{Mn}) \mathrm{As} / n-\mathrm{InGaAs}$ в величину общего сопротивления структуры. Установлено, что зависимость магнетосопротивления от внешнего магнитного поля имеет гистерезисный вид, особенности которого обусловлены влиянием напряжений растяжения в слое ( $\mathrm{Ga}, \mathrm{Mn}) \mathrm{As}$, выращенном поверх релаксированного буфера $\operatorname{In}_{x} \mathrm{Ga}_{1-x}$ As (содержание индия $x \approx 0.1$ ).

DOI: $10.21883 /$ FTP.2019.03.47287.9000

\section{1. Введение}

Разработка приборов спинтроники в настоящее время во многом зависит от достижений в области создания и исследования ферромагнитных полупроводников $[1,2]$. Современные методы формирования ферромагнитных полупроводников основаны на легировании материалов магнитными элементами, в роли которых, как правило, выступают переходные металлы ( $\mathrm{Mn}, \mathrm{Fe}, \mathrm{Cr}$ ); такие материалы называют разбавленными магнитными полупроводниками (РМП). „Классическим“ примером разбавленного магнитного полупроводника можно назвать полупроводники $\mathrm{A}^{\mathrm{III}} \mathrm{B}^{\mathrm{V}}$, легированные атомами $\mathrm{Mn}[1,3,4]$. В некоторых сплавах - например, (In, Mn)As и $(\mathrm{Ga}, \mathrm{Mn}) \mathrm{As})$ - марганец не только поставляет локализованные магнитные моменты, но и является акцептором, приводя к появлению дырок в валентной зоне.

На текущем уровне развития технологии разбавленных магнитных полупроводников можно выделить две основные тенденции. Первая связана с повышением температуры Кюри данных материалов (чаще всего температура Кюри ниже $300 \mathrm{~K})$. Для соединений $\left(\mathrm{A}^{\mathrm{III}}, \mathrm{Mn}\right) \mathrm{B}^{\mathrm{V}}$ прогресс в данном направлении с 2000-х годов незначителен, в то же время можно отметить работы по получению новых материалов на основе полупроводников $\mathrm{A}^{\mathrm{III}} \mathrm{B}^{\mathrm{V}}$, легированных атомами $\mathrm{Fe}$, которые сохраняют ферромагнитные свойства до комнатной температуры и выше [5,6].
Вторая тенденция в технологии РМП связана с созданием приборов спинтроники на их основе. В частности, в работах $[4,7,8]$ описано формирование и исследование свойств нелокальных спиновых клапанов приборов, принцип работы которых основан на эффекте инжекции спин-поляризованных носителей из намагниченного ферромагнитного слоя в полупроводник GaAs. Другим классом приборов спинтроники, включающих слои $\left(\mathrm{A}^{\mathrm{III}}, \mathrm{Mn}\right) \mathrm{B}^{\mathrm{V}}$, в основном $(\mathrm{Ga}, \mathrm{Mn}) \mathrm{As}$, являются $p-n$-диоды, в основу работы которых заложен принцип спин-зависимого транспорта носителей заряда в намагниченных слоях разбавленного магнитного полупроводника [9-11].

Следует отметить, что в отличие от нелокальных спиновых клапанов исследованию $p-n$-диодов со слоем $\left(\mathrm{A}^{\mathrm{III}}, \mathrm{Mn}\right) \mathrm{B}^{\mathrm{V}}$ в научной литературе посвящено небольшое количество публикаций. Так, в работах $[9,10]$ было изучено влияние магнитного упорядочения на электрический перенос в $p-n$-переходах со слоем GaMnAs или $\mathrm{GaMnN}$. Изготовленные $p-n$-переходы состояли из магнитных слоев, легированных $\mathrm{Mn}$, сформированных на немагнитных полупроводниковых подложках. Исследовались их электрические и магнитные свойства, которые показали, что обменное взаимодействие между носителями заряда и локализованными магнитными моментами атомов Mn оказывает значительное влияние на перенос заряда при температурах, близких к тем- 
пературе Кюри. Авторами [9] изучены магнитополевые зависимости вольт-амперных характеристик $p-n$-диодов для различных концентраций легирующей примеси в $p$ и $n$-областях. Это позволило провести анализ работы магнитных диодов как в обычном диффузионном, так и в туннельном режимах. В качестве p-областей диодов использовались однородные ферромагнитные тонкие пленки $\mathrm{Ga}_{1-x} \mathrm{Mn}_{x} \mathrm{As}(x=0.01-0.09)$ высокого кристаллического качества, изготовленные с использованием низкотемпературной $\left(200-300^{\circ} \mathrm{C}\right)$ молекулярнолучевой эпитаксии. Ожидалось, что намагничивание слоя ( $\mathrm{Ga}, \mathrm{Mn}) \mathrm{As}$ в направлении, совпадающем с направлением протекания тока, приведет к снижению последовательного сопротивления диода за счет уменьшения сопротивления слоя РМП (указанный эффект хорошо известен как отрицательное магнетосопротивление [4]).

Для магнитного диода $p^{+}-(\mathrm{Ga}, \mathrm{Mn}) \mathrm{As} / n-\mathrm{GaAs}$, включающего слабо легированный немагнитный слой $n$-GaAs (концентрация доноров $N_{D}=10^{17} \mathrm{~cm}^{-3}$ ) в области $p-n$-перехода, не было обнаружено отрицательного магнетосопротивления в температурном интервале $T=8-300 \mathrm{~K}$. Авторы [9] полагают, что затруднения с детектированием отрицательного магнетосопротивления связаны с отсутствием магнитного упорядочения в ( $\mathrm{Ga}, \mathrm{Mn}) \mathrm{As}$ в режиме смещения, соответствующем обеднению $p^{+}$-области $(\mathrm{Ga}, \mathrm{Mn}) \mathrm{As}$. В этом случае все компоненты тока, т.е. избыточный ток, ток рекомбинации и диффузионный ток, становятся независимыми от магнитного поля. В $p^{++}-n^{++}$-переходах $\mathrm{Ga}_{x} \mathrm{Mn}_{1-x} \mathrm{As} / \mathrm{GaAs}$, имеющих сильно легированные $p$ - и $n$-области, преобладал межзонный туннельный механизм протекания тока, при этом величина тока сильно зависела от магнитного упорядочения. В туннельном диоде при прямом смещении $\sim 300$ мВ (в этом режиме преобладает туннельный ток) наблюдалось большое по величине отрицательное магнетосопротивление при низких температурах (до $\sim 8 \%$ при $10 \mathrm{~K}$ ), которое насыщалось по мере увеличения внешнего магнитного поля до 4000 Э. Магнетосопротивление оставалось отрицательным до температур измерения $\sim 50 \mathrm{~K}$ (температура Кюри слоя GaMnAs). При больших напряжениях прямого смещения, когда в переносе преобладают диффузионный и избыточный токи, отрицательное магнетосопротивление не наблюдалось при любой температуре.

Структура (Ga, Mn)N-p-n-диода, исследованная в [10], была аналогичной рассмотренной в [9] структуре (Ga, Mn)As/GaAs, за исключением ферромагнитной легированной $\mathrm{Mn}$ области, которая была создана твердотельной диффузией и имела $n$-тип проводимости. Основной вывод работ $[9,10]$ состоял в том, что появление отрицательного магнетосопротивления в магнитных GaAs- $p-n$-диодах возможно только в случаях, когда обе стороны $p-n$-перехода сильно легированы и диод работает в туннельном режиме. В этом случае возникает спин-зависимый туннельный ток, который проявлялся в вольт-амперных характеристиках приборов при низких температурах как значительное уменьшение сопротивления структуры с увеличением магнитного поля.

Работа [11] представляет результаты изучения $p-i-n$ диодной структуры с квантовой ямой InGaAs/GaAs, coдержащей ферромагнитный слой GaMnAs в качестве полупроводника p-типа проводимости. Авторами показано, что внешнее магнитное поле приводит к уменьшению сопротивления слоя GaMnAs (на 5\% в магнитном поле $3600 Э$ при $10 \mathrm{~K}$ ). В режиме источника напряжения уменьшение сопротивления приводит к росту тока диода и, как следствие, к увеличению интенсивности электролюминесценции. При температуре, превышающей температуру Кюри GaMnAs, эффект уменьшался до нуля. Следует отметить, что здесь, в отличие от работ $[9,10]$, отрицательное магнетосопротивление наблюдалось для $p-i-n$-диода, а не для туннельного диода, причем эффект регистрировался при значениях тока, соответствующих режиму инжекции в область $p-n$-перехода.

Помимо экспериментальных исследований, в литературе представлен ряд теоретических работ, посвященных созданию моделей, описывающих эффекты, которые могут наблюдаться в магнитных $p-n$-переходах $[12,13]$. В цитированных работах предсказываются несколько явлений с возможными применениями в области спинтроники, в том числе спин-вольтаический эффект, эффект спинового клапана, экспоненциальное и гигантское магнетосопротивление.

В работе [14] приводится первое приближение к численному расчету, которое позволяет решить уравнения Пуассона и непрерывности для случая ферромагнитного и вырожденного $\left(p^{+}-n^{+}\right)$перехода. Поскольку он вырожден, носители в $p-n$-переходе могут рекомбинировать через прямой туннельный процесс из валентной зоны в зону проводимости и наоборот. Ферромагнетизм производит спиновое расщепление зоны проводимости в $n$-полупроводниках и валентной зоны в $p$-полупроводниках. Авторы [14] представляют расчет, который позволяет оценить асимметрию туннелирования для идеального перехода с произвольной спиновой поляризацией на каждой стороне и определяет зависимость тока от напряжения в области перехода. Полученные результаты предсказывают, что самое сильное влияние спиновой поляризации на величину тока происходит в области отрицательного дифференциального сопротивления.

Таким образом, согласно представленным литературным данным, вопросы спин-поляризованного транспорта в $p-n$-диодах вида ,разбавленный магнитный полупроводник/неферромагнитный полупроводник“ далеки от полного разрешения. Известны определенные конструкции диодов, содержащих $p-n$-переходы с ферромагнитными полупроводниковыми слоями, которые позволяют изучать процессы спин-зависимого переноса, но нет достаточного количества экспериментальных данных, которые бы позволили развить соответствующие теоретические представления.

В настоящей работе представлены результаты экспериментального изучения новой конструкции диодных 
гетероструктур с ферромагнитными слоями GaMnAs. Диодные структуры были изготовлены сочетанием методов МОС-гидридной эпитаксии (газофазной эпитаксии из металлоорганических соединений) и импульсного лазерного нанесения. В отличие от работ [9-13], были сформированы гетеропереходы (Ga, Mn)As/InGaAs; на указанных гетеропереходах был получен эффект отрицательного магнетосопротивления при температурах ниже точки Кюри (Ga, Mn)As.

\section{2. Методика эксперимента}

Структуры для исследований были сформированы комбинированным методом МОС-гидридной эпитаксии (МОСГЭ) и импульсного лазерного осаждения в едином ростовом цикле [15]. В качестве подложки были использованы пластины GaAs n-типа проводимости с ориентацией (001). Для испарения мишеней $\mathrm{Mn}$ и $\mathrm{GaAs}$ применялся лазер $\mathrm{Nd}: \mathrm{YAG}$ с длиной волны излучения 1.06 мкм, работающий в режиме модулированной добротности.

На нагретой до $T_{g}=650^{\circ} \mathrm{C}$ подложке $n$-GaAs (100) методом МОС-гидридной эпитаксии был сформирован буферный слой $\operatorname{In}_{x} \mathrm{Ga}_{1-x} \mathrm{As}$ (содержание индия $x \approx 0.1$ ) толщиной $\sim 1.8$ мкм, слабо легированный атомами $\mathrm{Si}$ (концентрация электронов $n \approx 8 \cdot 10^{16} \mathrm{~cm}^{-3}$ ). Затем температура роста понижалась до $330^{\circ} \mathrm{C}$ и путем поочередного лазерного распыления мишеней $\mathrm{Mn}$ и GaAs (соотношение времен распыления $t_{\mathrm{Mn}} / t_{\mathrm{GaAs}}=0.15$ ) был сформирован слой $(\mathrm{Ga}, \mathrm{Mn}) \mathrm{As} p$-типа проводимости толщиной $\sim 30$ и $\sim 50$ нм для структур 7912 и 7913 соответственно. Таким образом, формировались $p-i-n$-диоды, содержащие слой сильно легированного марганцем

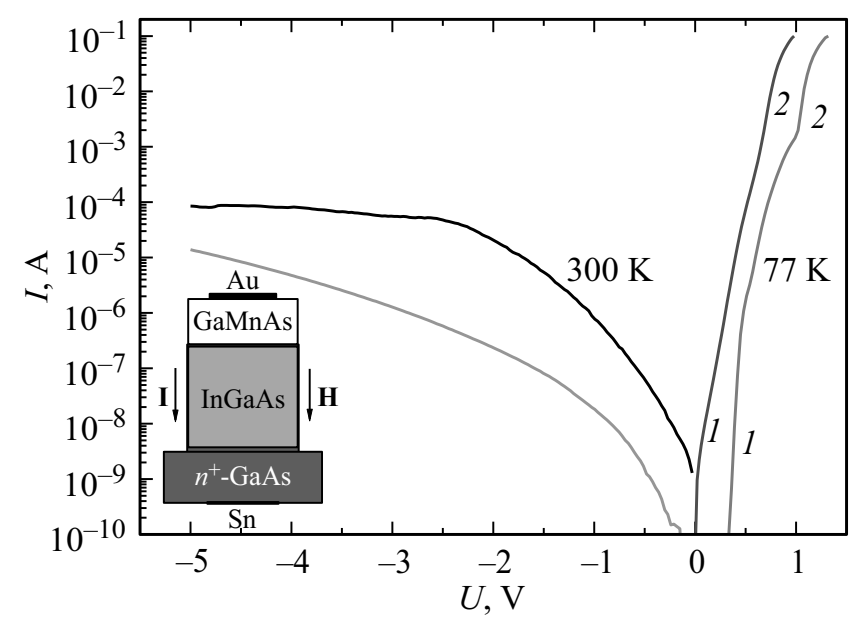

Рис. 1. Вольт-амперные характеристики диода 7913 при температурах $T=77$ и $300 \mathrm{~K} .1$ - участок малых токов в области небольшого по величине напряжения (до $500 \mathrm{MB}$ ), 2 - участок последовательного сопротивления (линейный участок ВАХ). На вставке - схема диода, показаны направления протекания тока в режиме прямого смещения и внешнего магнитного поля при измерении магнитополевых зависимостей.
$\mathrm{GaAs}$, a $i$-область представляет собой слабо легированный слой InGaAs. Схематическое изображение изготовленных диодных структур показано на вставке к рис. 1. Ранее было показано, что подобные слои GaMnAs обладают ферромагнитными свойствами при низких температурах [16,17].

Концентрация свободных носителей в буферном слое InGaAs определялась методом вольт-фарадных характеристик (BФХ) с использованием измерителя емкости EG\&G PAR-410 с частотой тестового сигнала 1 МГц при температурах 295 и $77 \mathrm{~K}$. Профили концентрации, полученные из ВФХ, показали однородное распределение легирующей примеси на глубинах $0.1-0.5$ мкм в обеих структурах. Значения концентрации свободных носителей при комнатной температуре для обеих структур составили $\sim(6-8) \cdot 10^{16} \mathrm{~cm}^{-3}$. Охлаждение до температуры $77 \mathrm{~K}$ практически не влияло на значения концентрации носителей, что говорит об отсутствии заметного количества глубоких уровней в структуре.

На поверхность образцов со стороны слоя (Ga, Mn)As наносился омический металлический контакт на основе $\mathrm{Au}$. Омический контакт к подложке $n^{+}$-GaAs формировался электроискровым вжиганием оловянной фольги. На последней стадии технологического процесса фотолитографическим методом и химическим травлением изготавливались меза-структуры диодов диаметром $\sim 600$ мкм.

В работе представлены результаты исследований вольт-амперных характеристик, в том числе в режиме приложения внешнего магнитного поля. Вольт-амперные характеристики (BAX) и магнетосопротивление диодных гетероструктур изучались с использованием источника-измерителя Keithley 2400 и гелиевого криостата замкнутого цикла Janis CCS-300S/202 при температурах от 10 до $300 \mathrm{~K}$. Внешнее магнитное поле величиной до 3600 Э прикладывалось в направлении, перпендикулярном поверхности структур, с помощью электромагнита. Магнитополевая зависимость сопротивления диодов изучалась в режимах обратного и прямого смещения.

\section{3. Результаты: характеризация структур}

Вольт-амперные характеристики $I(U)$ исследованного диода 7913, измеренные при температурах 300 и $77 \mathrm{~K}$, представлены на рис. 1. Характеристики диода 7912 подобны BAX для 7913, поэтому на рисунке они не представлены. Известно, что реальные ВАХ диодов отражают влияние таких факторов, как наличие поверхностных состояний на границе раздела слоев, генерация и рекомбинация носителей в обедненном слое, туннелирование носителей между состояниями в запрещенной зоне, которое обусловливает высокий уровень инжекции, наблюдаемый даже при небольшом смещении, и влияние последовательного сопротивления. 
Коэффициент неидеальности $n$ на участке малых токов в области небольшого по величине напряжения, до $500 \mathrm{MB}$ (участок 1) и значения последовательного сопротивления $R$ в области большого по величине напряжения, $>1.2 \mathrm{~B}$ (участок 2) для диода 7913

\begin{tabular}{c|c|c}
\hline Параметр & $300 \mathrm{~K}$ & $77 \mathrm{~K}$ \\
\hline$n$ & 1.70 & 1.9 \\
$R$, Ом & 2.2 & 2.3
\end{tabular}

Так, для исследованной структуры 7913 ток обратной ветки ВАХ при комнатной температуре медленно нарастает вплоть до $10^{-4} \mathrm{~A}$ с увеличением обратного напряжения до $5 \mathrm{~B}$. Подобное поведение обусловлено влиянием утечек через состояния на неоднородной границе раздела $(\mathrm{Ga}, \mathrm{Mn}) \mathrm{As} / n$-InGaAs (подобное поведение наблюдалось ранее на аналогичных структурах [18]). При прямом смещении ВАХ содержит несколько участков, которые были проанализированы: участок малых токов в области небольшого по величине напряжения (до $500 \mathrm{MB}$ ), обозначенный 1 , и участок 2 в области напряжений выше $1.2 \mathrm{~B}$, где ВАХ имеет линейный вид. Согласно [19], экспериментальные ВАХ в общем случае для участка 1 можно описать выражением

$$
I_{F} \propto \exp (q U / n k T) .
$$

Здесь коэффициент неидеальности $n=2$, если преобладает рекомбинационный ток, и $n=1$, если преобладает диффузионный ток. Если оба тока сравнимы по величине, то значение $n$ лежит в интервале от 1 до 2 [19]. Экспериментальные значения коэффициента неидеальности для диода 7913 были вычислены с использованием формулы (1) для ВАХ при 300 и $77 \mathrm{~K}$. Результаты вычислений представлены в таблице. Рассчитанные коэффициенты неидеальности принимают значения между 1 и 2, что свидетельствует о сопоставимой величине диффузионного и рекомбинационного токов.

Участок 2 в области $>1.2 \mathrm{~B}$, где ВАХ имеет линейный вид, отражает влияние последовательного сопротивления. Величина прямого смещения в этой области такова, что барьер практически отсутствует, а основной вклад в сопротивление дают монокристаллическая подложка GaAs и эпитаксиальные слои $(\mathrm{Ga}, \mathrm{Mn}) \mathrm{As}$ и InGaAs. Вычисленные по BAX значения сопротивления в данной области также представлены в таблице. Для обеих температур величина последовательного сопротивления не превышает 2.5 Ом.

В области прямых смещений $0.5<U<1.2$ В поведение ВАХ, по-видимому, определяется высоким уровнем инжекции. Согласно [19], при прямом смещении в условиях высокой плотности токов, когда концентрация инжектированных неосновных носителей сравнима по величине с концентрацией основных носителей, необходимо учитывать дрейфовую и диффузионную составляющие тока. Вследствие высокого уровня инжекции падение напряжения на $p-n$-переходе существенно меньше по сравнению с омическим падением в обеих областях диода.

\section{4. Результаты: влияние магнитного поля на вольт-амперные характеристики структур}

На рис. 2 представлены ВАХ диодов 7912 и 7913, измеренные при температуре $13 \mathrm{~K}$ и прямом смещении в отсутствие и при наличии магнитного поля. Как видно из представленных зависимостей, внешнее магнитное поле не оказывает заметного влияния на общий вид BAX, но влияет на величину токов (будет показано далее). Более подробные исследования были выполнены путем измерения магнитополевых зависимостей сопротивления диодов в режиме источника напряжения при нескольких значениях напряжения $0.9,1.0,1.1,1.2$ и 1.4 В (отмечено пунктиром на рис. 2). Согласно обсуждению ВАХ диода 7913 при 300 и $77 \mathrm{~K}$, эти величины прямого смещения можно отнести к участкам высокого уровня инжекции (от 0.9 до $1.2 \mathrm{~B}$ ) и последовательного сопротивления (выше 1.2В).

Результаты исследования магнетосопротивления при температуре измерений $13 \mathrm{~K}$ для диода 7913 (с толщиной слоя $\mathrm{GaMnAs} \sim 50$ нм) показаны на рис. 3 . Магнетосопротивление (MR) определялось как

$$
[R(H)-R(0)] / R(0),
$$

где $R(H)$ и $R(0)$ - значения сопротивления диода в магнитном поле $H$ и без магнитного поля. В качестве $R(0)$ и

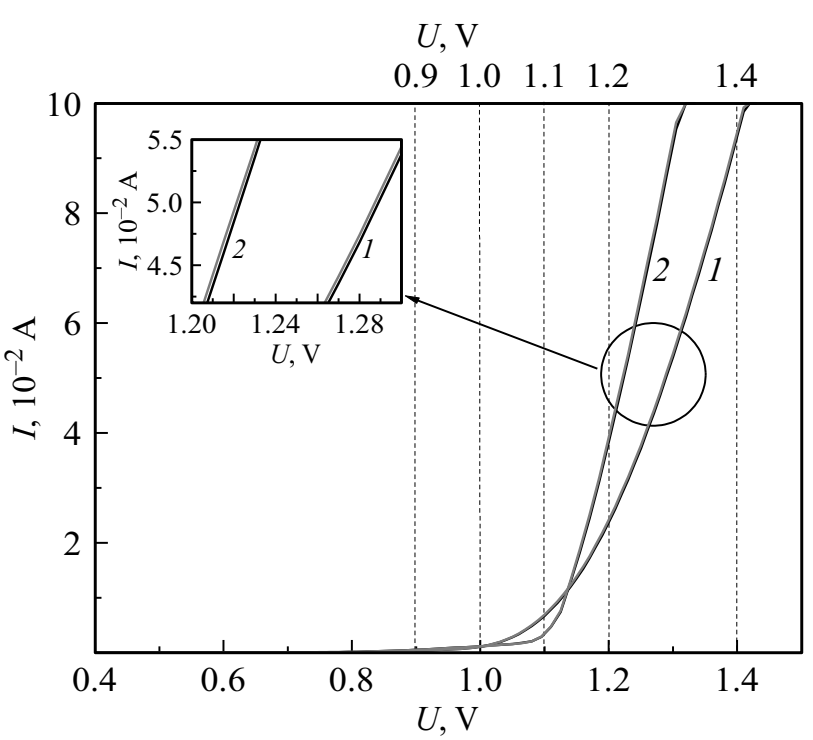

Рис. 2. Вольт-амперные характеристики диодов 7912 (1) и 7913 (2) при температуре $T=13 \mathrm{~K}$ и прямом смещении в отсутствие магнитного поля (черная линия) и в поле $3600 Э$ (серая линия). Пунктиром отмечены напряжения, при которых было выполнено изучение магнитополевых зависимостей сопротивления диодов в режиме источника напряжения (см. далее). 


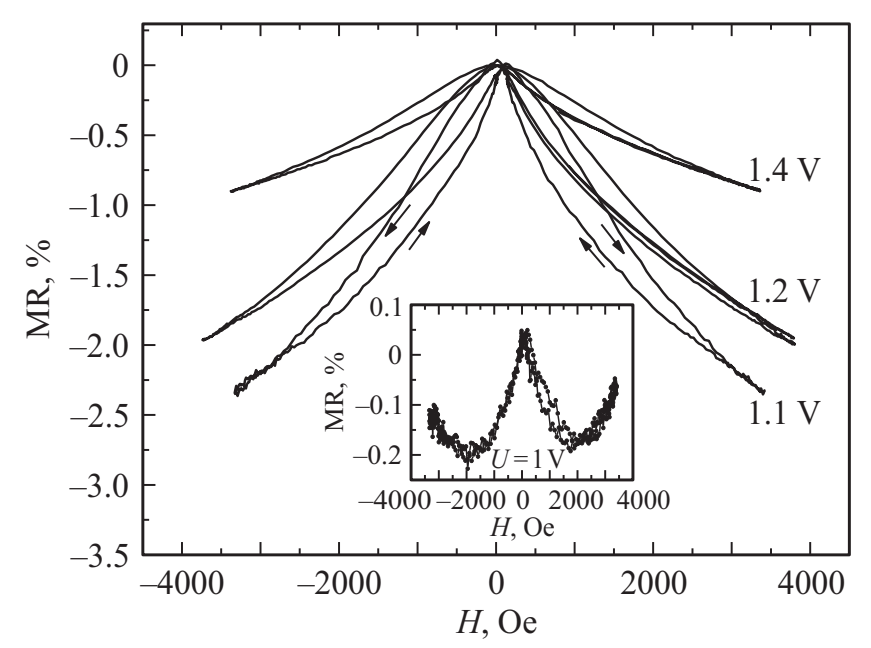

Рис. 3. Магнетосопротивление диодной структуры 7913 (толщина слоя GaMnAs 50 нм) при $T=13 \mathrm{~K}$ и различной величине прямого смещения (от 1.1 до $1.4 \mathrm{~B}$ ). На вставке - магнетосопротивление при напряжении $1 \mathrm{~B}$.

$R(H)$ брались статические значения сопротивления, т.е. отношение напряжения на диоде к току через диод,

$$
R(0, H)=U / I(0, H)
$$

причем напряжение на диоде не изменялось (измерения выполнялись в режиме источника напряжения), следовательно, изменение сопротивления связано с изменением тока, протекающего через диод в геометрии, показанной на вставке к рис. 1.

Видно, что при значениях смещения $>1.0$ В наблюдается отрицательное магнетосопротивление (ОМС) с гистерезисным характером зависимости от магнитного поля. Наличие отрицательного магнетосопротивления свидетельствует о влиянии магнитного упорядочения в слое $(\mathrm{Ga}, \mathrm{Mn}) \mathrm{As}$ на перенос спин-поляризованных носителей, в частности наблюдаемое уменьшение величины сопротивления диода 7913 в магнитном поле свидетельствует о повышении тока при заданном напряжении и связывается с уменьшением рассеяния спинполяризованных носителей заряда в магнитоупорядоченном слое [11]. Гистерезисный характер зависимостей на рис. 3 обусловлен преимущественной анизотропией магнитного упорядочения в направлении, перпендикулярном поверхности. Такая анизотропия возникает в результате напряжений растяжения, действующих на GaMnAs со стороны нижележащего эпитаксиального слоя InGaAs [17].

Магнетосопротивление диодной структуры 7912 (толщина слоя GaMnAs 30 нм) в зависимости от величины прямого смещения при температуре измерений $13 \mathrm{~K}$ показывает аналогичное поведение. Отличие состоит в том, что пороговое напряжение эффекта несколько ниже, а именно $U=0.9$ В. При меньших величинах подаваемого напряжения вклад $\mathrm{OMC}$ не регистрировался.
Для обеих структур наибольшее по модулю значение магнетосопротивления $\sim 2.3 \%$ в магнитном поле 3600 Э соответствует прямому смещению $U=1.1 \mathrm{~B}$. Сводная информация по эффекту отрицательного магнетосопротивления для исследованных структур представлена на рис. 4, где приведены зависимости максимального по модулю значения отрицательного магнетосопротивления от напряжения смещения на диоде для образцов 7912 и 7913. Обе зависимости являются немонотонными с максимумом при напряжении $1.1 \mathrm{~B}$. При напряжениях $U<0.9$ В эффект отрицательного магнетосопротивления не зарегистрирован, и при $U>1.1 \mathrm{~B}$ величина эффекта существенно снижается с ростом напряжения смещения. Статическое сопротивление диода $R$ экспоненциально уменьшается с увеличением $U$, о чем свидетельствуют зависимости $R(U)$, показанные на вставке к рис. 4.

Температурная зависимость эффекта отрицательного магнетосопротивления показана на рис. 5. Представлена эволюция кривых магнитополевой зависимости сопротивления с ростом температуры измерений для образца 7912. В случае образца 7913 были получены подобные результаты. Кривые измерены при величине прямого смещения $1.1 \mathrm{~B}$, для которого была получена максимальная величина эффекта ОМС. С увеличением температуры измерений величина ОМС (формула 2) монотонно спадает до $\sim 0.4 \%$ при $50 \mathrm{~K}$. На вставке к рис. 5 представлена зависимость модуля величины ОМС от температуры измерений в магнитном поле 3600 Э. Из приведенных данных видно, что эффект отрицательного магнетосопротивления сохраняется до температуры $50 \mathrm{~K}$, а выше $50 \mathrm{~K}$ стремится к нулю (так, при температуре $60 \mathrm{~K}$ величина ОМС составляет $\sim 0.1 \%$ ). Значение температуры $50 \mathrm{~K}$ хорошо согласуется с данными

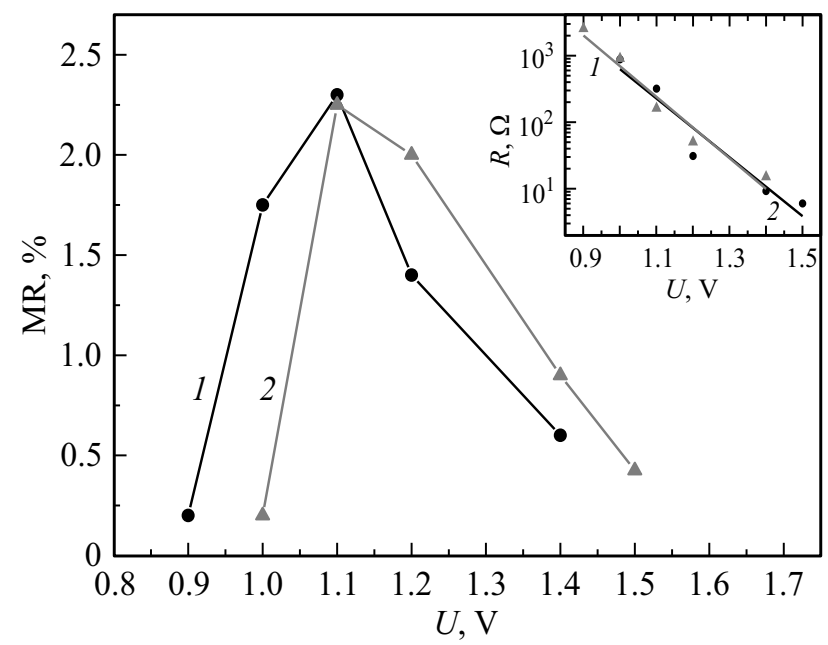

Рис. 4. Модуль магнетосопротивления (в магнитном поле 3600 Э) диодных структур 7912 (1) и 7913 (2) в зависимости от величины прямого смещения при температуре $13 \mathrm{~K}$. На вставке - зависимости статического сопротивления от напряжения смещения для структур 7912 (кружки) и 7913 (треугольники). 


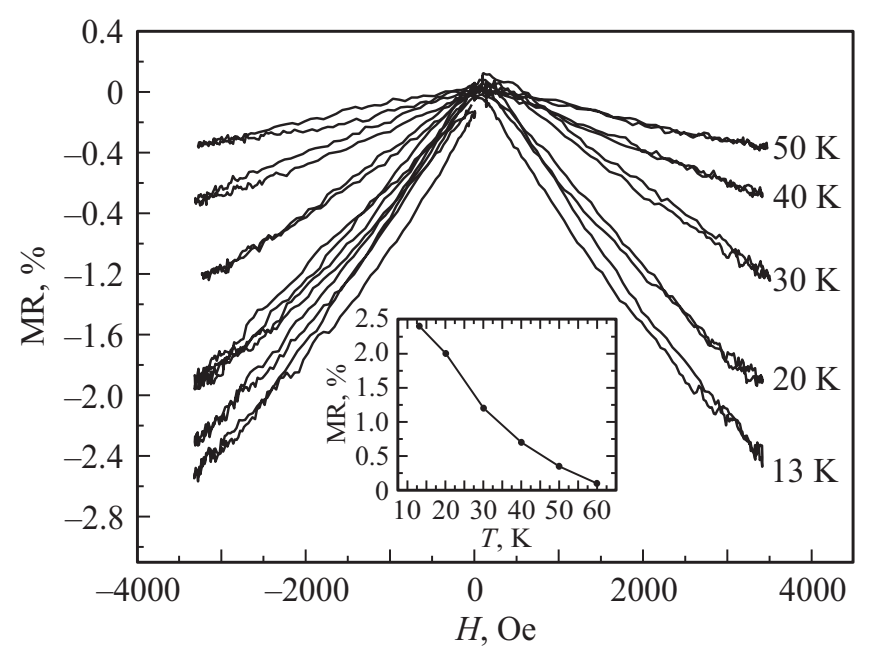

Рис. 5. Магнетосопротивление диодной структуры 7912 в зависимости от температуры при величине прямого смещения $1.1 \mathrm{~B}$. На вставке - температурная зависимость модуля MR в магнитном поле 3600 Э.

по температуре Кюри слоев (Ga, Mn)As, сформированных по аналогичной технологии [15-18]. Полученный результат позволяет сделать вывод о влиянии магнитных свойств слоя $(\mathrm{Ga}, \mathrm{Mn}) \mathrm{As}$ на зарегистрированный эффект отрицательного магнетосопротивления.

\section{5. Обсуждение результатов}

Как уже отмечалось ранее, образцы изучаемых диодов в качестве $p$-области содержат слой ферромагнитного полупроводника GaMnAs. Согласно опубликованным ранее данным [17], где представлено исследование влияния упругих напряжений на магнитные свойства эпитаксиальных слоев GaMnAs, полученных методом импульсного лазерного осаждения, температура фазового перехода ферромагнетик-парамагнетик (температура Кюри) GaMnAs составляет $\sim 50 \mathrm{~K}$. В работе [17] также показано, что в слоях GaMnAs, изготовленных на релаксированных буферных слоях $\operatorname{In}_{x} \mathrm{Ga}_{1-x}$ As (c напряжениями растяжения), магнитополевая зависимость намагниченности является нелинейной функцией с петлей гистерезиса при приложении магнитного поля перпендикулярно поверхности (что соответствует выбранной нами геометрии - вставка к рис. 1).

Поэтому полученные нами результаты исследований магнитополевой зависимости сопротивления мы связываем с влиянием намагничивания слоя ( $\mathrm{Ga}, \mathrm{Mn}) \mathrm{As}$ на общее сопротивление структуры. Последнее может быть приближенно представлено в виде суммы нескольких слагаемых:

$$
R_{p-n} \approx R_{(\mathrm{Ga}, \mathrm{Mn}) \mathrm{As}}+R_{\mathrm{InGaAs}}+R_{(n-\mathrm{GaAs})}+R_{C 1}+R_{C 2},
$$

где $R_{(\mathrm{Ga}, \mathrm{Mn}) \mathrm{As}}, R_{\mathrm{InGaAs}}, R_{(n-\mathrm{GaAs})}-$ сопротивления слоев (Ga, Mn)As, InGaAs и подложки GaAs соот- ветственно, $R_{C 1}-$ сопротивление гетероперехода (Ga, Mn)As/n-InGaAs, связанное с наличием разрыва зон, а также дефектных состояний на границе раздела, обеспечивающих дополнительное рассеяние, $R_{C 2}-$ сопротивление гетероперехода $n$-InGaAs $/ n$-GaAs, которое также связано с наличием разрыва зон и состояний на гетерогранице. Очевидно, что изменение сопротивления в присутствии магнитного поля за счет намагничивания слоя ( $\mathrm{Ga}, \mathrm{Mn}) \mathrm{As}$, показанное на рис. 4, может быть обусловлено только слагаемыми $R_{(\mathrm{Ga}, \mathrm{Mn}) \mathrm{As}}$ и $R_{C 1}[10,11,20]$.

При имеющихся концентрациях дырок в слое GaMnAs $\left(p \approx 6 \cdot 10^{19} \mathrm{~cm}^{-3}\right)$ и электронов в InGaAs $\left(n \approx 8 \cdot 10^{16} \mathrm{~cm}^{-3}\right.$ и $6 \cdot 10^{16} \mathrm{~cm}^{-3}$ для образцов 7912 и 7913 соответственно) бо́льшая часть обедненной области $p-n$-перехода будет расположена в слое InGaAs. Следовательно, в режиме обратного смещения и при малых напряжениях прямого смещения определяющий вклад в величину $R_{p-n}$ вносит слагаемое $R_{\mathrm{InGaAs}}-$ сопротивление слоя InGaAs, большая часть которого является обедненной. Это объясняет отсутствие эффекта отрицательного магнетосопротивления при обратном смещении диода, а также при малых прямых смещениях $(U<0.9 \mathrm{~B})$ : сопротивление слоя InGaAs не зависит от намагниченности (Ga, Mn)As. При повышении напряжения прямого смещения толщина обедненной области InGaAs уменьшается, а полное сопротивление $R_{\mathrm{InGaAs}}$ снижается и становится сравнимым с остальными величинами, входящими в формулу (4) для $R_{p-n}$. Напряжение, при котором вклады сопротивления $R_{\text {InGaAs }}$ и спинзависимых сопротивлений $R_{(\mathrm{Ga}, \mathrm{Mn}) \mathrm{As}}, R_{C 1}$ становятся сравнимыми, по-видимому, составляет $0.9-1.0$ В. При подаче прямого напряжения > $(0.9-1.0)$ В потенциальный барьер на границе ( $\mathrm{Ga}, \mathrm{Mn}) \mathrm{As} / n$-InGaAs практически полностью спрямляется, как следует из ВАХ, т.е. значительно уменьшается размер обедненной области в слое InGaAs.

Кроме этого, выравнивание величин $R_{\text {InGaAs }}$ и $R_{(\mathrm{Ga}, \mathrm{Mn}) \mathrm{As}}, R_{C 1}$ очень наглядно видно из кривой магнитополевой зависимости сопротивления, показанной для образца 7913 на вставке к рис. 3. При смещении $U=1.0$ В магнитополевая зависимость сопротивления состоит из двух областей. Сопротивление уменьшается для магнитного поля 0-2000Э и увеличивается при $H>2000$ Э. Первый участок связывается со снижением сопротивления в результате намагничивания слоя (Ga, Mn)As и, следовательно, с изменением слагаемых $R_{(\mathrm{Ga}, \mathrm{Mn}) \text { Аs }}$ и $R_{C 1}$. Второй участок связывается с повышением сопротивления в результате увеличения траектории носителей, протекающих в немагнитных слоях структуры (так называемое положительное магнетосопротивление) - т.е. с изменением сопротивления $R_{\text {InGaAs. }}$ Эффект положительного магнетосопротивления хорошо известен для немагнитных полупроводников и особенно заметно проявляется в материалах с высокой подвижностью [21]. Можно заключить, 
что при смещении, равном $1.0 \mathrm{~B}$, вклады уменьшения сопротивления ферромагнитного слоя ( $\mathrm{Ga}, \mathrm{Mn}) \mathrm{As}$ и увеличения сопротивления немагнитных слоев гетероструктуры сопоставимы по величине. В области низких магнитных полей доминирует „отрицательная“ составляющая, а в магнитном поле, соответствующем насыщению намагниченности ( $\mathrm{Ga}, \mathrm{Mn}) \mathrm{As}(H \approx 2000$ Э), больший вклад начинает вносить повышение сопротивления слоя InGaAs. Сопротивление подложки $n^{+}$-GaAs выведено из рассмотрения в силу того, что его значение вносит наименьший вклад в сумму (4). Действительно, с учетом уровня легирования подложки $\sim 10^{19} \mathrm{~cm}^{-3}$ и подвижности электронов $10^{3} \mathrm{~cm}^{2} / \mathrm{B} \cdot \mathrm{c}$ сопротивление подложки составляет $\sim 0.1$ Ом, что в 20 раз меньше минимального зарегистрированного сопротивления диода (2.2 Ом, см. таблицу). Сопротивление гетероперехода $n$-InGaAs/n-GaAs $R_{C 2}$ здесь также не рассматривается, поскольку его величина не зависит ни от магнитного поля, ни от условий электрического смещения и, таким образом, представляет собой некое постоянное слагаемое в сумме (4).

Таким образом, полученный в эксперименте эффект отрицательного магнетосопротивления обусловлен транспортом спин-поляризованных носителей в слое ( $\mathrm{Ga}, \mathrm{Mn}) \mathrm{As}$, а его величина зависит от изменения сопротивления этого слоя $R_{(\mathrm{Ga}, \mathrm{Mn}) \mathrm{As}}$ и сопротивления гетероперехода $R_{C 1}$. Вероятно, вклад указанных величин в общее сопротивление диода максимален при напряжении $U=1.1 \mathrm{~B}$. При напряжении $>1.1 \mathrm{~B}$, согласно ВАХ (рис. 2), происходит полное спрямление потенциальных барьеров в диоде. По мере увеличения напряжения на диоде величина модуля магнетосопротивления слоя ( $\mathrm{Ga}, \mathrm{Mn}) \mathrm{As}$ снижается вследствие увеличения доли неполяризованных по спину носителей в токопереносе через этот слой. Это объясняет общее уменьшение магнетосопротивления при увеличении напряжения на диоде выше $1.1 \mathrm{~B}$.

Наконец обсудим природу зависимости $R_{C 1}$ и $R_{(\mathrm{Ga}, \mathrm{Mn}) \mathrm{As}}$ от магнитного поля. Отрицательное магнетосопротивление для разбавленных магнитных полупроводников связано с уменьшением рассеяния при переносе спин-поляризованных носителей через магнитоупорядоченный материал $[3,4]$. Снижение сопротивления $R_{C 1}$ в магнитном поле связывается с расщеплением валентной зоны (Ga, $\mathrm{Mn}) \mathrm{As}$ при его намагничивании (величина расщепления оценивалась в работах $[3,4,20])$ и соответствующим уменьшением разрыва зон. Подобное расщепление зон приводит к снижению потенциального барьера для дырок на границе раздела (Ga, Mn)As/n-InGaAs, что эквивалентно уменьшению сопротивления $R_{C 1}$. Таким образом, вклад $R_{C 1}$ и $R_{(\mathrm{Ga}, \mathrm{Mn}) \mathrm{As}}$ в эффект отрицательного магнетосопротивления может быть аддитивен. Отметим, что разделение вкладов $R_{(\mathrm{Ga}, \mathrm{Mn}) \mathrm{As}}$ и $R_{C 1}$ затруднительно и не имеет практического смысла, поскольку для построения приборов на эффекте отрицательного магнетосопротивления наиболее важным представляется величина эффекта.

\section{6. Заключение}

Таким образом, на основании выполненного исследования диодных $p-i-n$-гетероструктур, содержащих $p$-слой ферромагнитного полупроводника GaMnAs, изготовленный методом импульсного лазерного нанесения, можно сделать следующие выводы:

- исследованные ВАХ диодов при 77 и $300 \mathrm{~K}$ позволяют заключить, что на участке малых токов в области небольшого по величине напряжения (до 500 мВ) вклады диффузионного и рекомбинационного токов сопоставимы;

- исследования электрических свойств диодов в магнитном поле, приложенном перпендикулярно плоскости $p-n$-перехода, обнаружили наличие отрицательного магнетосопротивления (при температурах меньше $50 \mathrm{~K}$ ), связанного с уменьшением рассеяния носителей заряда благодаря наличию ферромагнитного упорядочения, а также со снижением потенциального барьера на границе (Ga, Mn)As/ $n$-InGaAs;

- зависимость магнетосопротивления от внешнего магнитного поля имеет гистерезисный вид, особенности которого обусловлены влиянием напряжений растяжения в слое GaMnAs, выращенном поверх релаксированного буфера $\operatorname{In}_{x} \mathrm{Ga}_{1-x}$ As (содержание индия $x \approx 0.1$ );

- наблюдаемое отрицательное магнетосопротивление характеризуется немонотонной зависимостью от величины прямого смещения с максимумом $\sim 2.3 \%$ (внешнее магнитное поле 3600Э) в области напряжения, близкого к высоте потенциального барьера $p$-n-перехода; предположительно, наличие максимума связано с максимальным вкладом спин-зависимых сопротивления слоя ( $\mathrm{Ga}, \mathrm{Mn}) \mathrm{As}$ и сопротивления на границе ( $\mathrm{Ga}, \mathrm{Mn}) \mathrm{As} / n$-InGaAs в величину общего сопротивления структуры.

Работа выполнена в рамках реализации государственного задания, проект № 8.1751.2017/ПЧ Министерства образования и науки РФ, и при поддержке РФФИ (гранты № 17-37-80008_мол_эв_а, 16-07-01102_а). Работы, проведенные в ИПТМ РАН, выполнены в рамках государственного задания № 075-00475-19-00.

\section{Список литературы}

[1] M. Bououdina, Y. Song, S. Azzaza. https://doi.org/10.1016/B978-0-12-803581-8.02431-0

[2] M. Ciorga, A. Einwanger, U. Wurstbauer, D. Schuh, W. Wegscheider, D. Weiss. Phys. Rev. B, 79, 165321 (2009).

[3] F. Matsukura, H. Ohno, T. Dietl. In: Handbook of Magnetic Materials, vol. 14, ed. by K.H.J. Buschow (Elsevier, Amsterdam, 2002) p. 1.

[4] H. Ohno. J. Magn. Magn. Mater., 200, 110 (1999).

[5] A.V. Kudrin, Yu.A. Danilov, V.P. Lesnikov, M.V. Dorokhin, O.V. Vikhrova, D.A. Pavlov, Y.V. Usov, I.N. Antonov, R.N. Kriukov, A.V. Alaferdov, N.A. Sobolov. J. Appl. Phys., 122 (18), 183901 (2017). 
[6] N.T. Tu, P.N. Hai, L.D. Anh, M. Tanaka. Appl. Phys. Express, 11 (6), 063005 (2018).

[7] M. Ciorga, M. Schlapps, A. Einwanger, S. Geißler, J. Sadowski, W. Wegscheider, D. Weiss. New J. Phys., 9, 351 (2007).

[8] T. Kita, M. Kohda, Y. Ohno, F. Matsukura, H. Ohno. Phys. Status Solidi C, 3 (12), 4164 (2006).

[9] H. Holmberg, N. Lebedeva, S. Novikov, P. Kuivalainen, M. Malfait, V.V. Moshchalkov. Phys. Status Solidi A, 204 (3), 791 (2007).

[10] H. Holmberg, N. Lebedeva, S. Novikov, P. Kuivalainen, M. Malfait, V.V. Moshchalkov. IEEE Trans. Magnetics, 42 (10), 2712 (2006).

[11] А.В. Кудрин, М.В. Дорохин, Ю.А. Данилов, Е.И. Малышева. Письма ЖТФ, 37 (24), 57 (2011).

[12] I. Žutic, J. Fabian, S. Das Sarma. Phys. Rev. Lett., 88, 066603 (2002).

[13] J. Fabian, I. Zutic, S. Das Sarma. Phys. Rev. B, 66, 165301 (2002).

[14] E. Comesana, M. Aldegunde, G.A. Gehring, A.J. GarciaLoureiro. Proc. 2009 Spanish Conf. Electron Devices (Santiago de Compostela, 2009) p. 160.

[15] B.N. Zvonkov, O.V. Vikhrova, Yu.A. Danilov, P.B. Demina, M.V. Dorokhin, V.V. Podol'skii, E.S. Demidov, Yu.N. Drozdov, M.V. Sapozhnikov. J. Opt. Technol., 75, 389 (2008).

[16] Б.Н. Звонков, О.В. Вихрова, Ю.А. Данилов, П.Б. Демина, А.В. Кудрин, В.П. Лесников, В.В. Подольский. Нанотехника, № 1, 32 (2008).

[17] Б.Н. Звонков, О.В. Вихрова, Ю.А. Данилов, Ю.Н. Дроздов, А.В. Кудрин, М.В. Сапожников. ФТТ, 52 (11), 2124 (2010).

[18] M.V. Dorokhin, B.N. Zvonkov, Yu.A. Danilov, V.V. Podolskii, P.B. Demina, O.V. Vikhrova, E.I. Malysheva, M.V. Sapozhnikov. Int. J. Nanoscience, 6 (3), 221 (2007).

[19] С. Зи. Физика полупроводниковых приборов (М., Мир, 1984) т. 1

[20] J. Shiogai, M. Ciorga, M. Utz, D. Schuh, T. Arakawa, M. Kohda, K. Kobayashi, T. Ono, W. Wegscheider, D. Weiss, J. Nitta. Appl. Phys. Lett., 101, 212402 (2012).

[21] A.B. Pippard. Magnetoresistance in Metals (Cambridge University Press, 1989).

\section{The study of magnetic diodes with a GaMnAs layer produced by pulsed laser deposition}

B.N. Zvonkov' ${ }^{1}$ O.V. Vikhrova' ${ }^{1}$ Yu.A. Danilov ${ }^{1}$ M.V. Dorokhin ${ }^{1}$, A.V. Kudrin', I.L. Kalentyeva', E.A. Larionova1 , V.A. Kovalskiy' ${ }^{2}$, O.A. Soltanovich ${ }^{2}$

${ }^{1}$ Lobachevsky State University of Nizhny Novgorod, 603950 Nizhny Novgorod, Russia

2 Institute of Microelectronics Technology and High Purity Materials, Russian Academy of Sciences, 142432 Chernogolovka, Moscow region, Russia

Abstract The results of experimental study of new design diode heterostructures with ferromagnetic $(\mathrm{Ga}, \mathrm{Mn})$ As layers are presented. The diode structures were fabricated using a combination of MOCVD epitaxy and pulsed laser deposition and contained (Ga, Mn)As/n-InGaAs heterojunctions. The electrical properties of the diodes in the magnetic field applied perpendicular to the $p-n$-junction plane were investigated. The presence of negative magnetoresistance (at temperatures $<50 \mathrm{~K},(\mathrm{Ga}, \mathrm{Mn}$ )As Curie temperature), associated with decrease in carrier scattering due to ferromagnetic ordering and with decrease in the potential barrier at the $(\mathrm{Ga}, \mathrm{Mn}) \mathrm{As} / n$-InGaAs interface. The observed negative magnetoresistance demonstrated the non-monotonic dependence on the magnitude of the forward bias, with a maximum in the voltage range close to the height of the potential barrier of the $p-n$-junction. The presence of the maximum can be related to the determining contribution of the spin-dependent resistance of the (Ga, Mn)As layer and the resistance at the (Ga, Mn)As/n-InGaAs interface to the total resistance of the structure. It was established that the magnetoresistance dependence on the external magnetic field has a hysteresis form due to the influence of tensile stresses in the $(\mathrm{Ga}, \mathrm{Mn})$ As layer, grown over the relaxed $\operatorname{In}_{x} \mathrm{Ga}_{1-x}$ As buffer (indium content $x \approx 0.1$ ).

Редактор Л.В. Шаронова 